\title{
Resistance Profile of Aerobic Gram-Negative Bacilli: Study on Pseudomonas aeruginosa and Acinetobacter baumannii in a Tertiary Care Center
}

\author{
Hadi Younes, Laurene Haddad and Jacques Choucair* \\ Department of Infectious Diseases, Hôtel Dieu de France, Saint Joseph University, \\ Beirut, Lebanon \\ *Corresponding Author: Jacques Choucair, Department of Infectious Diseases, \\ Hôtel Dieu de France, Saint Joseph University, Beirut, Lebanon.
}

Received: February 25, 2020

Published: August 19, 2021

(C) All rights are reserved by Jacques

Choucair., et al.

\begin{abstract}
The natural selection has provided some resistance mechanisms of bacteria or adaptation face some antibiotiques. Or nowadays, overuse of antibiotics and exaggerated in inadequate conditions contributed to the increase in resistance rates. According to the World Health Organization, the resistance of bacteria to antibiotics is a major problem of the twenty-first century. Increasingly, bacteria acquire new resistance mechanisms and arrive to surpass the action of antibiotics. To fight against the proliferation of bacteria, it becomes necessary to find new and effective solutions to this major public health issue.
\end{abstract}

Keywords: Pseudomonas aeruginosa; Acinetobacter baumannii; Antibiotics

\section{Abbreviations}

PTZ: Piperacillin Tazobactam; CTZ: Ceftazidime; CFP: Cefepime; CIP: Ciprofloxacin; LEV: Levofloxacin; IMP: Imipenem; MER: Meronem; GEN: Gentamicin; AMK: Amikin; COL: Colistin

\section{Introduction}

Antibiotic resistance has become a major threat in current medical practice, especially in hospitalized patients. Pseudomonas aeruginosa and Acinetobacter baumannii are among the major germs responsible for health-care associated infections, with a high rate of multiresistant strains. The present study collected data between July and December 2019 regarding Pseudomonas aeruginosa and Acinetobacter baumannii resistance in a Middle Eastern tertiary care hospital. Infection site, age and sex of the patient and unit of hospitalization were described.

\section{Materials and Methods}

This is an observational retrospective study performed from July to December 2019 in a Middle Eastern tertiary care hospital.
All strains of Pseudomonas aeruginosa and Acinetobacter baumannii regardless of the site of infection were included. 281 cultures of Pseudomonas aeruginosa and 50 cultures of Acinetobacter baumannii were found. Antibiotic sensitivity for piperacillin-tazobactam, ceftazidime, cefepime, ciprofloxacin, levofloxacin, imipenem, meronem, amikacin, gentamicin and colistin were assessed. In addition, the unit of hospitalization, month of hospitalization and type of culture were listed.

\section{Results}

Numbers of Pseudomonas aeruginosa were highest in July and decreased progressively till December 2019. Similarly, cultures positive to A. baumannii were highest in July and lowest in December (Table 1). Extended drug resistant (XDR) P. aeruginosa were more common than multi drug resistant (MDR) strains throughout all the study. Prevalence of XDR and MDR types were highest in August and September respectively. For A. baumanii, MDR strains were only isolated in October and November. The highest number 
of XDR strains (8/50) were isolated in July (Table 2). In regular hospitalization units, 92/118 strains of $P$. aeruginosa were sensitive to piperacillin-tazobactam (78\%), 89 strains were sensitive to ceftazidime $(75.4 \%)$, and 90 strains were sensitive to cefepime $(76.3 \%)$.

$72 \%$ and $68.6 \%$ of $P$ aeruginosa strains were respectively sensitive to ciprofloxacin and levofloxacin, (85 strains for ciprofloxacin and 81 for levofloxacin) and $75.4 \%$ and $78.8 \%$ respectively sensitive to imipenem and meropenem, (89 strains for imipenem and 93 for meropenem). As for aminoglycosides, $83.1 \%$ and $86.4 \%$ of strains were sensitive respectively to gentamicin and amikacin, (98 strains for gentamicin and 102 for amikacin). Finally, 115 strains (97.5\%) were sensitive to a colistin.

Regarding the 22 strains of $A$. baumannii, for patients hospitalized on regular floors, 11 were sensitive to piperacillin-tazobactam, 9 were sensitive to ceftazidime, 12 were sensitive to cefepime, corresponding to $45.5 \%$ of resistance. 13 strains (59\%) were sensitive to quinolones (ciprofloxacin and levofloxacin), and 15 (68.2\%) of strains were sensitive to carbapenems (imipenem and meropenem). 12 and 13 strains were respectively sensitive to gentamicin and amikacin, Finally, 21/22 strains were sensitive to colistin, corresponding to a sensitivity level of $95.5 \%$.

In regular hospitalization wards, 92 strains of $P$. aeruginosa out of 118 were sensitive to piperacillin-tazobactam (78\%). 89 strains were sensitive to ceftazidime (75.4\%), and 90 strains were sensitive to cefepime (76.3\%). $72 \%$ and $68.6 \%$ of strains were respectively sensitive to ciprofloxacin and levofloxacin, (85 strains for ciprofloxacin and 81 for levofloxacin). $75.4 \%$ and $78.8 \%$ of strains were sensitive to imipenem and meropenem, respectively, (89 strains for imipenem and 93 for meropenem). $83.1 \%$ and $86.4 \%$ of strains were sensitive to gentamicin and amikacin respectively, (98 strains for gentamicin and 102 for amikacin). Finally, for colistin, 115 strains were sensitive, which corresponds to a sensitivity level of $97.5 \%$.

Among the 22 strains of $A$. baumannii, in the patients hospitalized on regular floors, 11 were sensitive to piperacillin-tazobactam, 9 were sensitive to ceftazidime. 12 strains were sensitive to cefepime, corresponding to $45.5 \%$ of resistance. 13 strains are sensitive versus 9 resistant to quinolones (ciprofloxacin and levofloxacin). 15 strains (68.2\%) are sensitive to carbapenems (imipenem and meropenem). $54.5 \%$ and $59.1 \%$ of strains were sensitive respectively to gentamicin and amikacin. Finally, 21 out of 22 strains were sensitive to colistin corresponding to a sensitivity level of 95.5\%.

In intensive care and critical care units, 46/64 strains of $P$. aeruginosa, were sensitive to piperacillin-tazobactam (71.9\%), 44 strains were sensitive to ceftazidime and cefepime (68.8\%), 73.4\% and $65.6 \%$ respectively sensitive to ciprofloxacin and levofloxacin, (47 for ciprofloxacin and 42 for levofloxacin). 50\% and $73.4 \%$ of the strains were respectively sensitive to imipenem and meropenem, (32 strains for imipenem and 47 for meropenem). $82.8 \%$ and $81.3 \%$ of the strains were sensitive to gentamicin and amikacin respectively, (53 for gentamicin and 52 for amikacin). Regarding co-

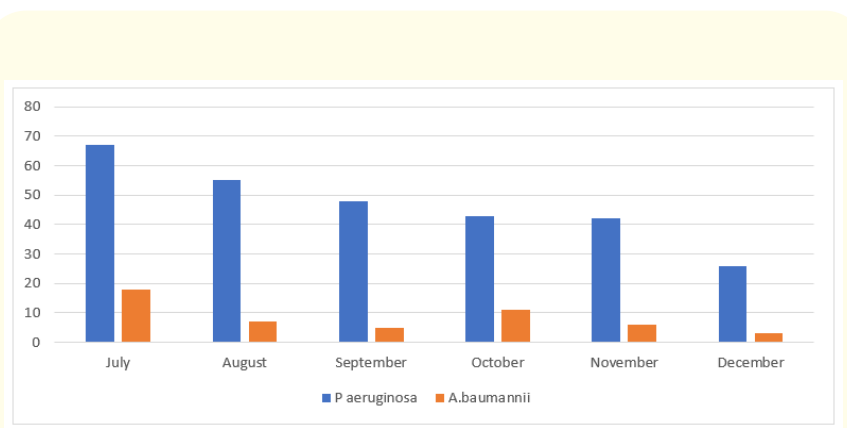

Graph 1: Distribution of P. aeruginosa and A. baumannii cultures according to month of hospitalization.

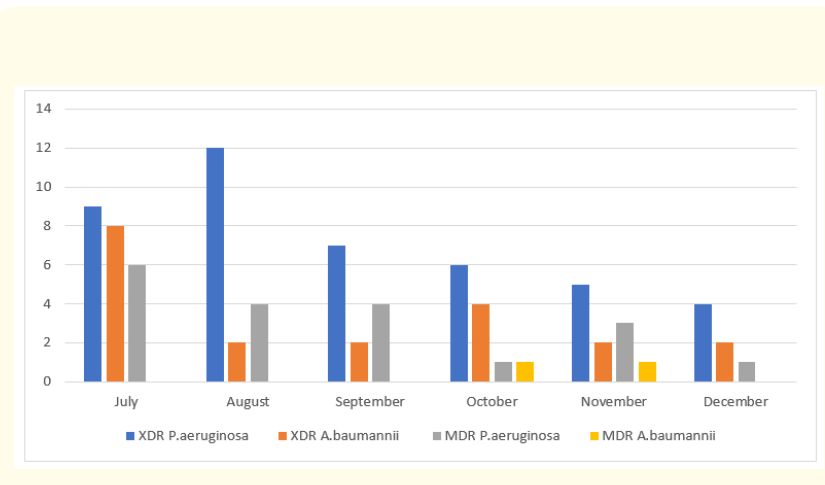

Graph 2: Distribution of $P$. aeruginosa and $A$. baumannii XDR and MDR according to month of hospitalization. 


\begin{tabular}{|l|c|c|c|c|}
\hline & P aeruginosa & P aeruginosa & A baumanii & A baumanii \\
\hline & $\begin{array}{c}\text { Sensitive } \\
\text { strains, } \%\end{array}$ & $\begin{array}{c}\text { Resistant } \\
\text { strains, } \%\end{array}$ & $\begin{array}{c}\text { Sensitive } \\
\text { strains, } \%\end{array}$ & $\begin{array}{c}\text { Resistant } \\
\text { strains, } \%\end{array}$ \\
\hline PTZ & $92 ; 78 \%$ & $26 ; 22 \%$ & $11 ; 50 \%$ & $11 ; 50 \%$ \\
\hline CTZ & $89 ; 75 \%$ & $29 ; 24 \%$ & $9 ; 41 \%$ & $13 ; 59 \%$ \\
\hline CFP & $90 ; 76 \%$ & $28 ; 24 \%$ & $12 ; 54.5 \%$ & $10 ; 45 \%$ \\
\hline CIP & $85 ; 72 \%$ & $33 ; 28 \%$ & $13 ; 59 \%$ & $9 ; 41 \%$ \\
\hline LEV & $81 ; 68.6 \%$ & $37 ; 31,4 \%$ & $13 ; 59 \%$ & $9 ; 41 \%$ \\
\hline IMP & $89 ; 75.4 \%$ & $29 ; 24.6 \%$ & $15 ; 68 \%$ & $7 ; 32 \%$ \\
\hline MER & $93 ; 79 \%$ & $25 ; 21 \%$ & $15 ; 68 \%$ & $7 ; 32 \%$ \\
\hline GEN & $98 ; 83 \%$ & $20 ; 17 \%$ & $12 ; 54.5 \%$ & $10 ; 45.5 \%$ \\
\hline AMK & $102 ; 86.4 \%$ & $16 ; 13.6 \%$ & $13 ; 59 \%$ & $9 ; 41 \%$ \\
\hline COL & $115 ; 97.5 \%$ & $3 ; 2.5 \%$ & $21 ; 95.5 \%$ & $1 ; 4.5 \%$ \\
\hline
\end{tabular}

cin, imipenem, meropenem, and gentamicin. Regarding amikacin, all 7 strains of $A$. baumannii were resistant. 6 out of 7 strains were sensitive to colistin, corresponding to an $85.7 \%$ sensitivity rate.

In ER patients, among the 57 strains of P. aeruginosa, 48 were sensitive to piperacillin-tazobactam (84.2\%), 49 were sensitive to ceftazidime (86\%). 40 strains were sensitive to cefepime (80.7\%). For ciprofloxacin and levofloxacin, respectively, $70.2 \%$ and $63.2 \%$ of strains were sensitive ( 40 strains for ciprofloxacin and 36 for levofloxacin). To imipenem and meropenem, respectively, $89.5 \%$ and $91.2 \%$ of strains were sensitive (51 strains for imipenem and 52 for meropenem). To gentamicin and amikacin, respectively, 93\% and $94.7 \%$ of strains were sensitive (53 strains for gentamicin and 54 for amikacin). For colistin, all strains were sensitive.

Table 1: Resistance profile of $P$. aeruginosa and A. baumannii to different antibiotics in regular floor patients during last six months of 2019.

Among the 9 strains of $A$. baumannii in ER patients, 6 were sensitive to piperacillin-tazobactam (66.7\%), 6 were sensitive to ceftazidime (66.7\%), 5 to cefepime (54.4\%). $66.7 \%$ of the strains were sensitive to quinolones (ciprofloxacin and levofloxacin) as well as $66.7 \%$ of strains were sensitive to carbapenems (imipenem and meropenem). $66.7 \%$ and $55.6 \%$ of strains were sensitive respectively to gentamicin and amikacin ( 6 for gentamicin and 5 for amikacin). All 9 strains were sensitive to colistin.

\begin{tabular}{|l|c|c|c|c|}
\hline & P aeruginosa & P aeruginosa & A baumannii & A baumannii \\
\hline & $\begin{array}{c}\text { Sensitive } \\
\text { strains, } \%\end{array}$ & $\begin{array}{c}\text { Resistant } \\
\text { strains, } \%\end{array}$ & $\begin{array}{c}\text { Sensitive } \\
\text { strains, } \%\end{array}$ & $\begin{array}{c}\text { Resistant } \\
\text { strains, } \%\end{array}$ \\
\hline PTZ & $46 ; 72 \%$ & $18 ; 28 \%$ & $1 ; 14 \%$ & $6 ; 86 \%$ \\
\hline CTZ & $44 ; 69 \%$ & $20 ; 31 \%$ & $1 ; 14 \%$ & $6 ; 86 \%$ \\
\hline CFP & $44 ; 69 \%$ & $20 ; 31 \%$ & $1 ; 14 \%$ & $6 ; 86 \%$ \\
\hline CIP & $47 ; 73,4 \%$ & $17 ; 26,6 \%$ & $1 ; 14 \%$ & $6 ; 86 \%$ \\
\hline LEV & $42 ; 65,6 \%$ & $22 ; 34,4 \%$ & $1 ; 14 \%$ & $6 ; 86 \%$ \\
\hline IMP & $32 ; 50 \%$ & $32 ; 50 \%$ & $1 ; 14 \%$ & $6 ; 86 \%$ \\
\hline MER & $47 ; 73,4 \%$ & $17 ; 26,6 \%$ & $1 ; 14 \%$ & $6 ; 86 \%$ \\
\hline GEN & $53 ; 83 \%$ & $11 ; 17 \%$ & $1 ; 14 \%$ & $6 ; 86 \%$ \\
\hline AMK & $52 ; 81 \%$ & $12 ; 19 \%$ & $9 ; 82 \%$ & $2 ; 18 \%$ \\
\hline COL & $63 ; 98,4 \%$ & $1 ; 1,6 \%$ & $6 ; 86 \%$ & $1 ; 14 \%$ \\
\hline
\end{tabular}

listin, 63 out of 64 strains were sensitive, corresponding to a $98.4 \%$ sensitivity level.

Among the 7 strains of $A$. baumannii, only one strain was sensitive to piperacillin-tazobactam in intensive care and critical care Table 2: Resistance profile of $P$. aeruginosa and A. baumannii to different antibiotics used in intensive care and critical care units during the last six months of 2019. 


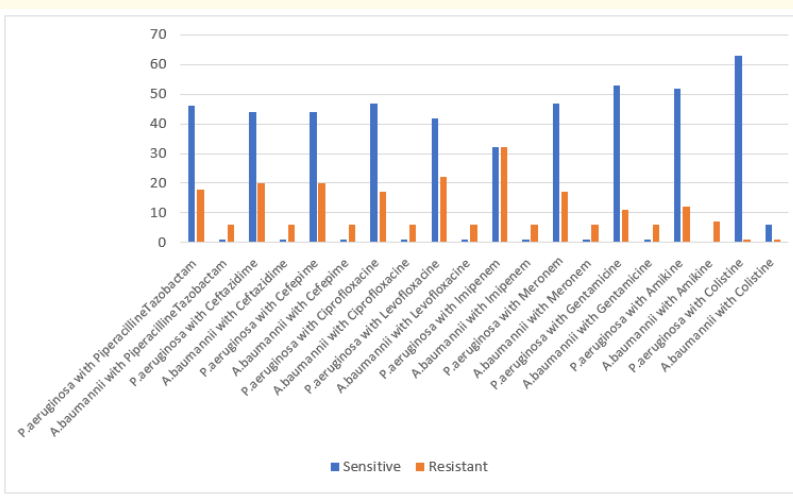

Graph 4: Graph showing the number and pattern of resistance of $P$. aeruginosa and $A$. baumannii to the ten different antibiotics used in intensive care and in the medical resuscitation unit of the Hôtel Dieu de France in the last six months of 2019.

\begin{tabular}{|l|c|c|c|c|}
\hline & P aeruginosa & P aeruginosa & A baumannii & A baumannii \\
\hline & $\begin{array}{c}\text { Sensitive } \\
\text { strains, \% }\end{array}$ & $\begin{array}{c}\text { Resistant } \\
\text { strains, \% }\end{array}$ & $\begin{array}{c}\text { Sensitive } \\
\text { strains, \% }\end{array}$ & $\begin{array}{c}\text { Resistant } \\
\text { strains, \% }\end{array}$ \\
\hline PTZ & $48 ; 84 \%$ & $9 ; 16 \%$ & $6 ; 67 \%$ & $3 ; 33 \%$ \\
\hline CTZ & $49 ; 86 \%$ & $8 ; 14 \%$ & $6 ; 67 \%$ & $3 ; 33 \%$ \\
\hline CFP & $46 ; 81 \%$ & $11 ; 19 \%$ & $6 ; 67 \%$ & $3 ; 33 \%$ \\
\hline CIP & $40 ; 70 \%$ & $17 ; 30 \%$ & $6 ; 67 \%$ & $3 ; 33 \%$ \\
\hline LEV & $36 ; 63 \%$ & $21 ; 37 \%$ & $6 ; 67 \%$ & $3 ; 33 \%$ \\
\hline IMP & $51 ; 89,5 \%$ & $6 ; 10,5 \%$ & $6 ; 67 \%$ & $3 ; 33 \%$ \\
\hline MER & $52 ; 91 \%$ & $5 ; 9 \%$ & $6 ; 67 \%$ & $3 ; 33 \%$ \\
\hline GEN & $53 ; 93 \%$ & $4 ; 7 \%$ & $6 ; 67 \%$ & $3 ; 33 \%$ \\
\hline AMK & $53 ; 94 \%$ & $4 ; 7 \%$ & $5 ; 55,6 \%$ & $4 ; 44,4 \%$ \\
\hline COL & $57 ; 100 \%$ & $0 ; 0 \%$ & $9 ; 100 \%$ & $0 ; 0 \%$ \\
\hline
\end{tabular}

Table 3: Resistance profile of P. aeruginosa and A. baumannii to different antibiotics used in ER patients during the last six months of 2019.

In the outpatient setting, among 38 strains of P. aeruginosa, 31 were sensitive to piperacillin-tazobactam (81.6\%), 27 were sensitive to ceftazidime $(71.1 \%), 28$ were sensitive to cefepime $(73.7 \%)$. 17 (44.7\%) and 16 (42.61\%) of strains were sensitive respectively to ciprofloxacin and levofloxacin. 28 strains (73.7\%) were sensitive to carbapenems (imipenem and meropenem). 31 (81.6\%) and 32

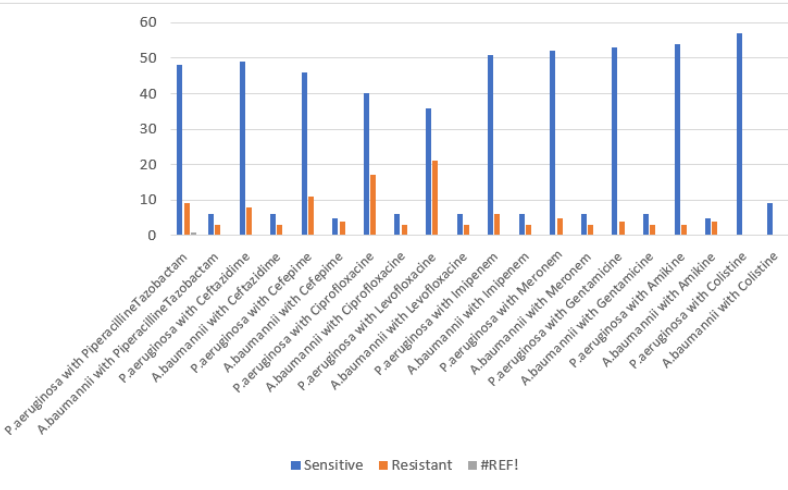

Graph 5: Graph showing the number and pattern of resistance of $P$. aeruginosa and $A$. baumannii to the ten different antibiotics used in the emergency room of the Hôtel Dieu de France in the last six months of 2019.

(84.2\%) of strains were sensitive respectively to gentamicin and amikacin. Finally, all 38 strains were sensitive for colistin.

Among the 11 strains of $A$. baumannii found in the outpatients, 9 were sensitive to piperacillin-tazobactam (81.8\%), 8 strains were sensitive to ceftazidime $(72.7 \%), 9$ strains were sensitive to cefepime $(81.8 \%)$. Nine strains were equally sensitive to ciprofloxacin, levofloxacin, imipenem, meropenem, gentamicin and amikacin. Finally, all the 11 strains were sensitive for colistin.

\begin{tabular}{|l|c|c|c|c|}
\hline AB & P aeruginosa & P aeruginosa & A baumannii & A baumannii \\
\hline & $\begin{array}{c}\text { Sensitive } \\
\text { strains, } \%\end{array}$ & $\begin{array}{c}\text { Resistant } \\
\text { strains, } \%\end{array}$ & $\begin{array}{c}\text { Sensitive } \\
\text { strains, } \%\end{array}$ & $\begin{array}{c}\text { Resistant } \\
\text { strains, } \%\end{array}$ \\
\hline PTZ & $31 ; 81,6 \%$ & $7 ; 18,4 \%$ & $9 ; 82 \%$ & $2 ; 18 \%$ \\
\hline CTZ & $27 ; 71 \%$ & $11 ; 29 \%$ & $8 ; 73 \%$ & $3 ; 27 \%$ \\
\hline CFP & $28 ; 74 \%$ & $10 ; 26 \%$ & $9 ; 82 \%$ & $2 ; 18 \%$ \\
\hline CIP & $17 ; 45 \%$ & $21 ; 55 \%$ & $9 ; 82 \%$ & $2 ; 18 \%$ \\
\hline LEV & $16 ; 42 \%$ & $22 ; 58 \%$ & $9 ; 82 \%$ & $2 ; 18 \%$ \\
\hline IMP & $28 ; 74 \%$ & $10 ; 26 \%$ & $9 ; 82 \%$ & $2 ; 18 \%$ \\
\hline MER & $28 ; 74 \%$ & $10 ; 26 \%$ & $9 ; 82 \%$ & $2 ; 18 \%$ \\
\hline GEN & $31 ; 81,6 \%$ & $7 ; 18,4 \%$ & $9 ; 82 \%$ & $2 ; 18 \%$ \\
\hline AMK & $32 ; 84 \%$ & $6 ; 16 \%$ & $9 ; 82 \%$ & $2 ; 18 \%$ \\
\hline COL & $38 ; 100 \%$ & $0 ; 0 \%$ & $11 ; 100 \%$ & $0 ; 0 \%$ \\
\hline
\end{tabular}

Table 4: Resistance profile of $P$. aeruginosa and A. baumannii to different antibiotics used in out patients during the last six months of 2019. 


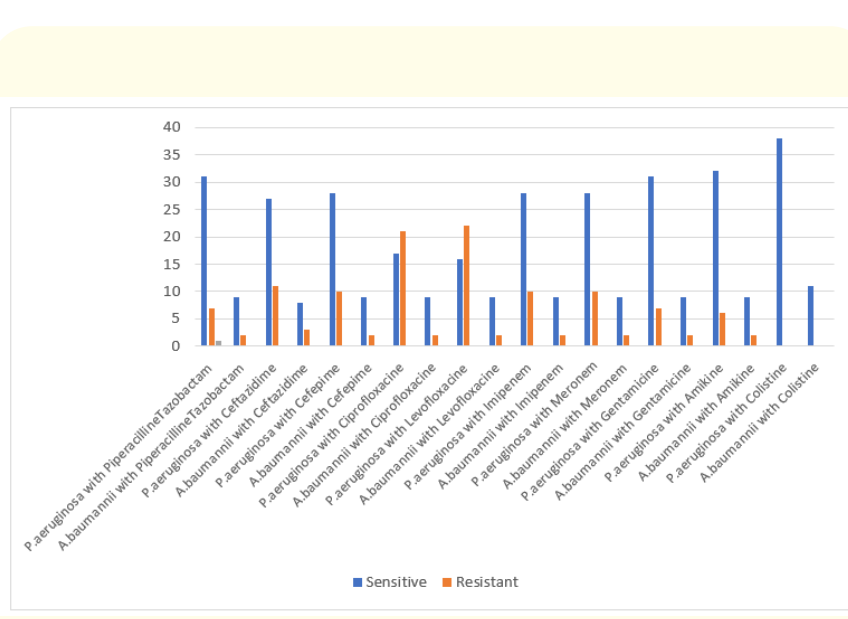

Graph 6: Graph showing the number and pattern of resistance of $P$. aeruginosa and $A$. baumannii to the ten different antibiotics used as outpatient at the Hôtel Dieu de France in the last six months of 2019.
In summary, for P. aeruginosa and A. baumannii respectively, the sensitivity to antibiotics is presented here, according to the unit of admission of the patient (intensive care or critical care unit, regular floor, ER and outpatient).

As for the infection site, for a total of 33 P. aeruginosa found in bloodstream or in catheters, $23(69.7 \%)$ strains were sensitive to piperacillin-tazobactam, $22(66.7 \%)$ to ceftazidime, $22(66.7 \%)$ to cefepime. $25(75.8 \%)$ and $23(69.7 \%)$ strains were respectively sensitive to ciprofloxacin and levofloxacin.

$15(45.5 \%)$ and 19 (57.6\%) of strains were sensitive to imipenem and meronem respectively. 27 (81.8\%) and 26 (78.1\%) of strains were sensitive to gentamicin and amikacin respectively. Finally, all 33 strains were sensitive to colistin.

For a total of 10 A. baumannii found in bloodstream or catheters, 5 (50\%) strains were sensitive to piperacillin-tazobactam, 3 strains (30\%) were sensitive to ceftazidime and cefepime. 5 strains (50\%)

\begin{tabular}{|l|c|c|c|c|c|c|c|c|}
\hline & \multicolumn{2}{|c|}{ Regular floor } & \multicolumn{2}{c|}{ ICU } & \multicolumn{2}{c|}{ ER } & \multicolumn{2}{c|}{ Outpatients } \\
\hline & Sensitive & Resistant & Sensitive & Resistant & Sensitive & Resistant & Sensitive & Resistant \\
\hline $\begin{array}{l}\text { Piperacillin } \\
\text { Tazobactam }\end{array}$ & $78.0 \%$ & $22.0 \%$ & $71.9 \%$ & $28.1 \%$ & $84.2 \%$ & $15.8 \%$ & $81.6 \%$ & $18.4 \%$ \\
\hline Ceftazidime & $75.4 \%$ & $24.6 \%$ & $68.8 \%$ & $31.3 \%$ & $86.0 \%$ & $14.0 \%$ & $71.1 \%$ & $28.9 \%$ \\
\hline Cefepime & $76.3 \%$ & $23.7 \%$ & $68.8 \%$ & $31.3 \%$ & $80.7 \%$ & $19.3 \%$ & $73.7 \%$ & $26.3 \%$ \\
\hline Ciprofloxacin & $72.0 \%$ & $28.0 \%$ & $73.4 \%$ & $26.6 \%$ & $70.2 \%$ & $29.8 \%$ & $44.7 \%$ & $55.3 \%$ \\
\hline Levofloxacin & $68.6 \%$ & $31.4 \%$ & $65.6 \%$ & $34.4 \%$ & $63.2 \%$ & $36.8 \%$ & $42.1 \%$ & $57.9 \%$ \\
\hline Imipenem & $75.4 \%$ & $24.6 \%$ & $50.0 \%$ & $50.0 \%$ & $89.5 \%$ & $10.5 \%$ & $73.7 \%$ & $26.3 \%$ \\
\hline Meronem & $78.8 \%$ & $21.2 \%$ & $73.4 \%$ & $26.6 \%$ & $91.2 \%$ & $8.8 \%$ & $73.7 \%$ & $26.3 \%$ \\
\hline Gentamicin & $83.1 \%$ & $16.9 \%$ & $82.8 \%$ & $17.2 \%$ & $93.0 \%$ & $7.0 \%$ & $81.6 \%$ & $18.4 \%$ \\
\hline Amikin & $86.4 \%$ & $13.6 \%$ & $81.3 \%$ & $18.8 \%$ & $94.7 \%$ & $5.3 \%$ & $84.2 \%$ & $15.8 \%$ \\
\hline Colistin & $97.5 \%$ & $2.5 \%$ & $98.4 \%$ & $1.6 \%$ & $100.0 \%$ & $0.0 \%$ & $100.0 \%$ & $0.0 \%$ \\
\hline
\end{tabular}

\begin{tabular}{|l|c|c|}
\hline Floor vs ER & Floor vs Outpatient & ER vs Outpatient \\
\hline P-value 0.00228 & P-value 0.00092 & P-value 0.00006 \\
\hline
\end{tabular}

Table 5: Table showing the percentage of resistance and susceptibility to different $P$. aeruginosa antibiotics as outpatient and intrahospital (care, emergency and stages) at the HDF.

Citation: Jacques Choucair., et al. "Resistance Profile of Aerobic Gram-Negative Bacilli: Study on Pseudomonas aeruginosa and Acinetobacter baumannii in a Tertiary Care Center". Acta Scientific Microbiology 4.9 (2021): 137-146. 


\begin{tabular}{|l|c|c|c|c|c|c|c|c|}
\hline & \multicolumn{2}{|c|}{ Floor } & \multicolumn{2}{c|}{ ICU } & \multicolumn{2}{c|}{ Urgences } & Outpatient \\
\hline & Sensible & Resistant & Sensible & Resistant & Sensible & Resistant & Sensible & Resistant \\
\hline $\begin{array}{l}\text { Piperacillin } \\
\text { Tazobactam }\end{array}$ & $50.0 \%$ & $50.0 \%$ & $14.3 \%$ & $85.7 \%$ & $66.7 \%$ & $33.3 \%$ & $81.8 \%$ \\
\hline Ceftazidime & $40.9 \%$ & $59.1 \%$ & $14.3 \%$ & $85.7 \%$ & $66.7 \%$ & $33.3 \%$ & $72.7 \%$ & $27.3 \%$ \\
\hline Cefepime & $54.5 \%$ & $45.5 \%$ & $14.3 \%$ & $85.7 \%$ & $55.6 \%$ & $44.4 \%$ & $81.8 \%$ & $18.2 \%$ \\
\hline Ciprofloxacin & $59.1 \%$ & $40.9 \%$ & $14.3 \%$ & $85.7 \%$ & $66.7 \%$ & $33.3 \%$ & $81.8 \%$ & $18.2 \%$ \\
\hline Levofloxacin & $59.1 \%$ & $40.9 \%$ & $14.3 \%$ & $85.7 \%$ & $66.7 \%$ & $33.3 \%$ & $81.8 \%$ & $18.2 \%$ \\
\hline imipenem & $68.2 \%$ & $31.8 \%$ & $14.3 \%$ & $85.7 \%$ & $66.7 \%$ & $33.3 \%$ & $81.8 \%$ & $18.2 \%$ \\
\hline Meronem & $68.2 \%$ & $31.8 \%$ & $14.3 \%$ & $85.7 \%$ & $66.7 \%$ & $33.3 \%$ & $81.8 \%$ & $18.2 \%$ \\
\hline Gentamicin & $54.5 \%$ & $45.5 \%$ & $14.3 \%$ & $85.7 \%$ & $66.7 \%$ & $33.3 \%$ & $81.8 \%$ & $18.2 \%$ \\
\hline Amikacin & $59.1 \%$ & $40.9 \%$ & $0.0 \%$ & $100.0 \%$ & $55.6 \%$ & $44.4 \%$ & $81.8 \%$ & $18.2 \%$ \\
\hline Colistin & $95.5 \%$ & $4.5 \%$ & $85.7 \%$ & $14.3 \%$ & $100.0 \%$ & $0.0 \%$ & $100.0 \%$ & $0.0 \%$ \\
\hline
\end{tabular}

\begin{tabular}{|l|c|c|}
\hline Floor vs ER & Floor vs outpatient & ER vs outpatient \\
\hline P-value 0.00843 & P-value 0.00015 & P-value 0.00474 \\
\hline
\end{tabular}

Table 6: Table showing the percentage of resistance and susceptibility to the different antibiotics of A. baumannii as outpatient and intrahospital (care, emergency and floor) at the HDF hospital.

\begin{tabular}{|l|c|c|c|c|}
\hline Antibiotic & Sensitive & Resistant & Sensitive & Resistant \\
\hline $\begin{array}{l}\text { Piperacillin-Tazo- } \\
\text { bactam }\end{array}$ & 23 & 10 & $69.7 \%$ & $30.3 \%$ \\
\hline Ceftazidime & 22 & 11 & $66.7 \%$ & $33.3 \%$ \\
\hline Cefepime & 22 & 11 & $66.7 \%$ & $33.3 \%$ \\
\hline Ciprofloxacin & 25 & 8 & $75.8 \%$ & $24.2 \%$ \\
\hline Levofloxacin & 23 & 10 & $69.7 \%$ & $30.3 \%$ \\
\hline Imipenem & 15 & 18 & $45.5 \%$ & $54.5 \%$ \\
\hline Meronem & 19 & 14 & $57.6 \%$ & $42.4 \%$ \\
\hline Gentamicin & 27 & 6 & $81.8 \%$ & $18.2 \%$ \\
\hline Amikin & 26 & 7 & $78.8 \%$ & $21.2 \%$ \\
\hline Colistin & 33 & 0 & $100.0 \%$ & $0.0 \%$ \\
\hline
\end{tabular}

Table 7: Number of $P$. aeruginosa isolated in the blood and catheters for the last six months and their resistance profile to different antibiotics.

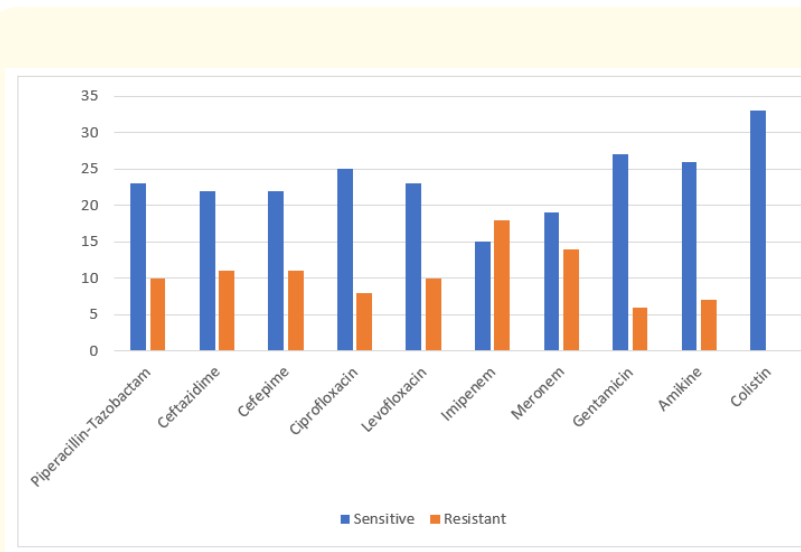

Graph 7: Chart showing the number of $P$. aeruginosa isolated in blood and catheters over the last six months at HDF with their resistance profile to different antibiotics. were sensitive to ciprofloxacin as well as for levofloxacin. $6(60 \%)$ of strains were sensitive to imipenem and to meropenem. 4 (40\%) of strains were sensitive to gentamicin and to amikacin, Finally, 9 (90\%) were sensitive for colistin. 


\begin{tabular}{|l|c|c|c|c|}
\hline Antibiotic & Sensitive & Resistant & Sensitive & Resistant \\
\hline $\begin{array}{l}\text { Piperacilline } \\
\text {-Tazobactam }\end{array}$ & 5 & 5 & $50.0 \%$ & $50.0 \%$ \\
\hline Ceftazidime & 3 & 7 & $30.0 \%$ & $70.0 \%$ \\
\hline Cefepime & 3 & 7 & $30.0 \%$ & $70.0 \%$ \\
\hline Ciprofloxacin & 5 & 5 & $50.0 \%$ & $50.0 \%$ \\
\hline Levofloxacin & 5 & 5 & $50.0 \%$ & $50.0 \%$ \\
\hline Imipenem & 6 & 4 & $60.0 \%$ & $40.0 \%$ \\
\hline Meronem & 6 & 4 & $60.0 \%$ & $40.0 \%$ \\
\hline Gentamicin & 4 & 6 & $40.0 \%$ & $60.0 \%$ \\
\hline Amikin & 4 & 6 & $40.0 \%$ & $60.0 \%$ \\
\hline Colistin & 9 & 1 & $90.0 \%$ & $10.0 \%$ \\
\hline
\end{tabular}

Table 8: Number of A. baumannii isolated in the blood or the catheters among last six months with their resistance profile to different antibiotics.

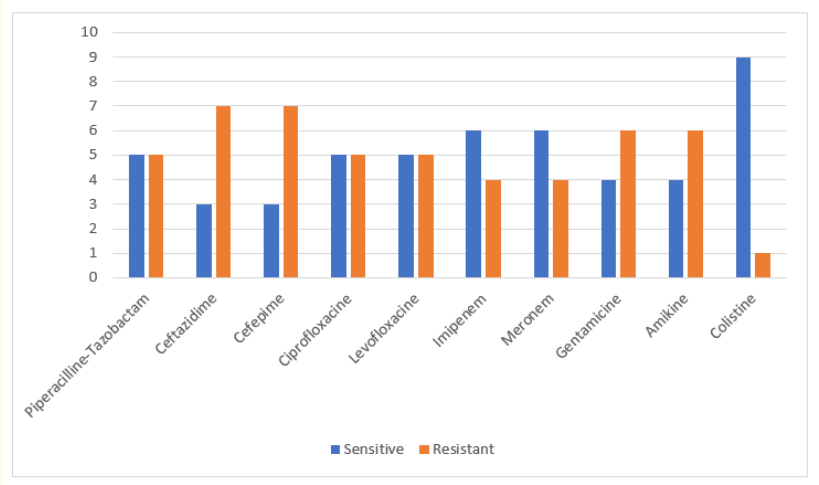

Graph 8: Graph showing the number of $A$. baumannii isolated in blood and catheters in the last six months at HDF with their resistance profile to different antibiotics.

\section{For $P$, aeruginosa}

Among the 281 strains isolated, 16 (5.7\%) were resistant to ceftazidime but sensitive to piperacillin-tazobactam. There were 90 (32\%) strains resistant to ciprofloxacin, 79 (28\%) resistant to imipenem, and 39 (14\%) resistant to amikacin.

\section{For A. baumannii}

Among the 50 collected strains of $A$. baumannii, 19 (38\%) were

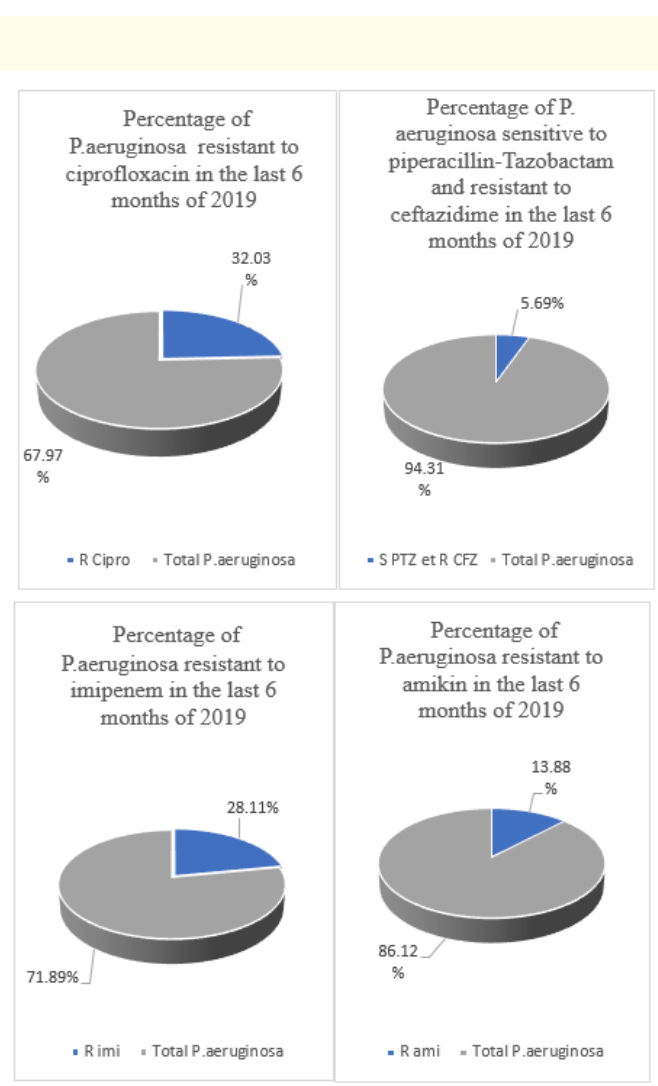

Figure 1

resistant to imipenem, 23 (46\%) resistant to amikacin, and 2 (4\%) to colistin.

\section{Discussion}

Considering the month of the patients' hospitalization, we note that the number of $P$. aeruginosa and $A$. baumannii infections decreases between July and December. This parallels the studies previously done in the United States during 8 years among 132 hospitals that showed that the frequency of gram-negative infections increased during summer and dropped in winter [1]. Another study done in 1996 had similarly showed an increase by more than $50 \%$ of A. baumannii and P. aeruginosa infection rates during summer months [2]. Warmth and humidity could contribute to an increased proliferation of $P$. aeruginosa and $A$. baumannii in the environment, which in turns increase the infection rate of these pathogens [3]. Increased temperature is thought to increase the 
Percentage of A. baumannii resistant to imipenem in the last 6 months of 2019

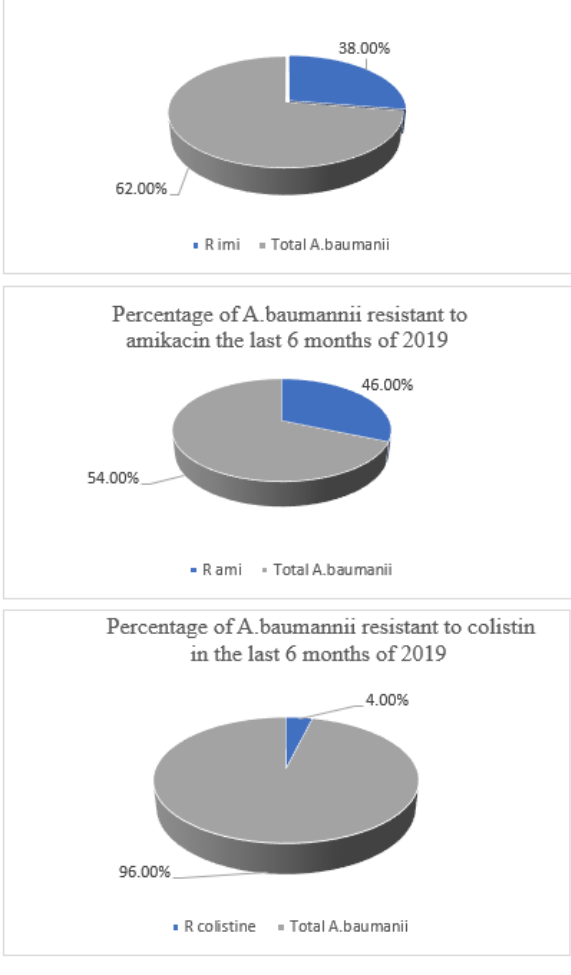

Figure 2 expression of the A lipid fraction of the lipopolysaccharide of the external membrane of these bacterial agents. This could be associated to a higher virulence rate in these germs [4] thereby increasing infection incidence and virulence during summer.

It would therefore be important to increase hygiene measures, especially hand washing, restriction of public drinking water and restriction of the use of carbapenems in empirical treatment, in order to limit this increase in the rate of infections with $P$. aeruginosa and $A$. baumannii, especially during summer [5].

In addition, in our hospitalization floors, we note that Pseudomonas aeruginosa is mainly susceptible to piperacillin-tazobactam (78\%), more than ceftazidime (75.4\%), carbapenems (75.4\%) and more than ciprofloxacin (72\%). This is in line with studies carried out at the public hospital in Ceará, Brazil, where a high percentage of resistance to carbapenems (about 42\%) was observed compared to other antibiotics [6]. Another study carried out between 2004 and 2008 showed an increase in the rate of resistance to carbapenems from $6 \%$ to $20 \%$ [7], as well as another study done in China in 2013 [8]. All this favors and justifies the strategy of restricting the use of carbapenems even in oncology service, this being because of the increasing percentage of carbapenemases producing enterobacteriales according to studies carried out in 2016 in 301 patients with a long stay in hospital in New York [9].

As for medical and surgical ICUs, we found in our study that Pseudomonas aeruginosa was $73.4 \%$ sensitive to meropenem, $72 \%$ to piperacillin-tazobactam, $69 \%$ to ceftazidime and 50\% to imipenem. Note that the resistance rates of Acinetobacter were much higher than for Pseudomonas for all antibiotics including also colistin (1.6\% for Pseudomonas against $14.3 \%$ for Acinetobacter). This is in line with a study done in 2017 in Brazil that had shown higher resistance rates for Acinetobacter compared to pseudomonas, with colistin resistance of $12 \%$ for Acinetobacter [10]. This would likely be due to Acinetobacter's ability to survive for a long time, especially in intensive care units (in the medical equipment used, gloves, beds, through hand contact) [11] and the high prevalence of bla OXA-23 mutants responsible for resistance to carbapenems and piperacillin. [12]. In addition, the numbers of pseudomonas and Acinetobacter resistant to amikacin and gentamicin in hospitals, whether on the floors or in the intensive care, are 14 to $17 \%$ for pseudomonas and $46 \%$ for Acinetobacter. In addition, Acinetobacter has a resistance of about $4 \%$ to colistin. These percentages are quite alarming for molecules considered to be broad-spectrum antibiotics with vital use as combination therapy or in septic shock and febrile neutropenia. This requires the reduction of prescribing aminoglycosides, especially amikacin as single dose, in the treatment of sepsis without recommendations, pending the results and favoring monotherapies when justified as to the guidelines.

For strains in emergency room patients, which normally represent community infections, the sensitivity of pseudomonas was significantly better compared with that of hospitalized patients except for quinolones, where the resistance reached 44\% (probably due to excessive over the counter consumption of quinolones in the community whereas community acquired Acinetobacter resistance rates to quinolones are lower than in hospital. These community Acinetobacter infections would be a major cause of se-

Citation: Jacques Choucair., et al. "Resistance Profile of Aerobic Gram-Negative Bacilli: Study on Pseudomonas aeruginosa and Acinetobacter baumannii in a Tertiary Care Center". Acta Scientific Microbiology 4.9 (2021): 137-146. 
vere pneumonia with a high mortality rate. Several factors would increase the risk of these community Acinetobacter infections such as smoking, alcoholism, diabetes mellitus and chronic obstructive pulmonary disease [13].

In addition, we also find that the sensitivity of pseudomonas outside of the hospital is almost similar to that of strains in the ER, with a rather sensitive profile. On the other hand, Acinetobacter strains outside of hospital care have a much better sensitization profile than those in the emergency room and on the floors (with a sensitivity of $81.6 \%$ ). This could be explained by a probable source difference between the strains of outpatients and those hospitalized.

Finally, for bacteremia and catheters infections, according to our study, pseudomonas is best treated with piperacillin-tazobactam, cephalosporins, aminoglycosides and colistin. These antibiotics would be better than carbapenems since they have a resistance rate of $54 \%$ to imipenem and $42 \%$ to meropenem. This is in line with the Study for Monitoring Antimicrobial Resistance Trends (SMART) done between 2002 and 2011 that showed a resistance rate to imipenem of 20 to $40 \%$ [14].

\section{Conclusion}

In conclusion, this study revealed several important facts for the empirical management of infected patients both in the regular floors, in intensive care units, and in the emergency or community.

We note first that the number of infections with $P$ aeruginosa and $A$. baumannii increases in summer and falls in winter.

In addition, the strains of Pseudomonas aeruginosa in outpatients are almost similar to those retrieved in the emergency room, with a rather sensitive profile: $P$. aeruginosa is most sensitive to piperacillin-tazobactam, more than ciprofloxacin, ceftazidime and carbapenems. In the medical and surgical intensive care units, we noticed that Pseudomonas aeruginosa was sensitive to piperacillintazobactam and ceftazidime but with a higher sensitivity to meropenem compared to the imipenem.

In addition, outpatient Acinetobacter baumannii strains have a better sensitivity profile than the hospitalized and emergency room strains.
A restriction on the use of carbapenems should be considered in order to reduce the selection pressure of resistant bacteria, except for bacteremia and Acinetobacter catheter infections where carbapenems are the treatment of choice.

Finally, the high resistance figures of Pseudomonas and Acinetobacter to aminoglycosides and especially to amikacin justify the importance of reducing the prescription of aminoglycosides in case of sepsis in single doses without recommendations pending the results. It would therefore be more effective in these cases to promote monotherapies when justified in the guidelines.

\section{Bibliography}

1. Eber MR., et al. "Seasonal and temperature-associated increases in gram-negative bacterial bloodstream infections among hospitalized patients". PLoS One 6.9 (2011): e25298.

2. McDonald LC., et al. "Seasonal variation of Acinetobacter infections: 1987-1996. Nosocomial Infections Surveillance System". Clinical Infectious Diseases 29 (1999): 1133-1137.

3. Perencevich EN., et al. "Summer peaks in the incidences of Gram-negative bacterial infection among hospitalized patients". Infection Control and Hospital Epidemiology 29 (2008): 1124-1131.

4. Trent MS. "Biosynthesis, transport, and modification of lipid A". Biochemistry and Cell Biology 82.1 (2004): 71-86.

5. Maragakis LL and Perl TM. "Acinetobacter baumannii: epidemiology, antimicrobial resistance, and treatment options". Clinical Infectious Diseases 46.8 (2008): 1254-1263.

6. Barros LM., et al. "Prevalência de micro-organismos e sensibilidade antimicrobiana de infecções hospitalares em unidade de terapia intensiva de hospital público no Brasil". Revista de Ciências Farmacêuticas Básica e Aplicada 33.3 (2012): 429-435.

7. Baumgart AMK., et al. "Prevalence of carbapenem resistant Pseudomonas aeruginosa and Acinetobacter baumannii in high complexity hospital". Brazilian Journal of Infectious Diseases 14.5 (2010): 433-436.

8. Xu J., et al. "Surveillance and Correlation of Antimicrobial Usage and Resistance of Pseudomonas aeruginosa: A Hospital Population-Based Study". PLoS ONE 8.11 (2013): e78604. 
9. Nishant Prasad., et al. "Asymptomatic rectal colonization with carbapenem-resistant Enterobacteriaceae and Clostridium difficile among residents of a long-term care facility in New York City". American Journal of Infection Control 44.5 (2016): 525532.

10. Otávio Hallal., et al. "Carbapenem-resistant Acinetobacter baumannii contamination in an intensive care unit". Revista da Sociedade Brasileira de Medicina Tropical 50.2 (2017): 167-172.

11. Dijkshoorn L., et al. "An increasing threat in hospitals: multidrug-resistant Acinetobacter baumannii". Nature Reviews Microbiology 5.12 (2007): 939-951.

12. Mugnier PD., et al. "Worldwide dissemination of the bla OXA-23 carbapenemase gene of Acinetobacter baumannii". Emerging Infectious Diseases 16.1 (2010): 35-40.

13. Anstey NM., et al. "Community-acquired Acinetobacter pneumonia in the Northern Territory of Australia". Clinical Infectious Diseases 14 (1992): 83.

14. Morrissey I., et al. "A Review of Ten Years of the Study for Monitoring Antimicrobial Resistance Trends (SMART) from 2002 to 2011". Pharmaceuticals (Basel) 6 (2013): 1335.

15. Lim SK., et al. "The outcomes of using colistin for treating multidrug resistant Acinetobacter species bloodstream infections". Journal of Korean Medical Science 26 (2011): 325.

\section{Volume 4 Issue 9 September 2021}

(C) All rights are reserved by Jacques Choucair., et al. 